\title{
Temperature Dependent Magnon-Phonon Coupling in bcc Fe from Theory and Experiment
}

\author{
F. Körmann, ${ }^{1}$ B. Grabowski, ${ }^{1}$ B. Dutta,${ }^{1}$ T. Hickel,${ }^{1}$ L. Mauger, ${ }^{2}$ B. Fultz,${ }^{2}$ and J. Neugebauer ${ }^{1}$ \\ ${ }^{1}$ Max-Planck-Institut für Eisenforschung GmbH, D-40237 Düsseldorf, Germany \\ ${ }^{2}$ California Institute of Technology, W. M. Keck Laboratory 138-78, Pasadena, California 91125, USA
}

(Received 18 May 2014; published 14 October 2014)

\begin{abstract}
An $a b$ initio based framework for quantitatively assessing the phonon contribution due to magnonphonon interactions and lattice expansion is developed. The theoretical results for bcc Fe are in very good agreement with high-quality phonon frequency measurements. For some phonon branches, the magnonphonon interaction is an order of magnitude larger than the phonon shift due to lattice expansion, demonstrating the strong impact of magnetic short-range order even significantly above the Curie temperature. The framework closes the previous simulation gap between the ferro- and paramagnetic limits.

An understanding of the mutual interaction between different temperature-induced excitations in solids is a pivotal challenge for the simulation of thermodynamic properties of many materials. A particularly important interaction is the delicate interplay between magnetic and atomic degrees of freedom, building the basis for many methodological applications [disordered local moments (DLM) molecular dynamics [1], magnetic empirical potentials [2]] as well as for integrated computational materials engineering (magnetic shape memory alloys [3], Ni-based superalloys [4], steels [5,6]). For Fe-based materials, it is well known that magnetoelastic effects are of tremendous importance for the interpretation of various phenomena such as the Invar effect [7], diffusion processes [8], dislocation dynamics [9], and phase stabilities [10].

Experimental studies of phonons at elevated temperatures can help elucidate magnon-phonon coupling. Neutron scattering experiments of phonon dispersions have provided important data at selected temperatures [11]. Nuclear resonant inelastic $\mathrm{x}$-ray scattering measurements are more amenable for showing thermal trends with measurements at many temperatures, and we have recently performed nuclear resonant inelastic $\mathrm{x}$-ray scattering measurements of the phonon density of states of bcc Fe at 38 temperatures through the Curie transition [12]. Nonharmonic changes in the phonon DOS and vibrational entropy were found to track the change in magnetization with temperature. Since experimental analysis of phonon DOS broadening suggests explicit anharmonic contributions from phonon-phonon interactions to be an order of magnitude smaller, these new results are suggestive of large magnon-phonon interactions in bcc Fe.

Parameter-free electronic structure calculations like density functional theory (DFT) in principle provide access to interatomic forces, spin-polarized energetics, and their interactions. Force-constant calculations and spin simulations have indeed been performed for decades $[10,13]$. However, most studies have been restricted to separate investigations of the two effects, whereas their mutual coupling could only be addressed in recent years $[1,14-16]$. The $T=0 \mathrm{~K}$ limit of a ferromagnetic system like $\mathrm{Fe}$ is the most straightforward case [17] since calculating force constants for a single magnetic configuration with all spins pointing in the same direction is sufficient ("FM limit" in Fig. 1). The infinite-temperature limit of a paramagnetic system with fully disordered spins ("PM limit") is significantly more challenging due to the large magnetic phase space that needs to be sampled for an accurate prediction of the coupling. Significant progress has been made only very recently with techniques based on DLM and spin molecular dynamics $[1,18]$, a spin-spiral approach [15], dynamical mean field theory [14], and a spin-space averaging procedure [16].

Given the complexity of the problem, present day methods are currently applied only at very low temperatures $(T \approx 0 \mathrm{~K}$ ), where significant long-range order is present, and at very high temperatures $\left(T \gg T_{C} ; T_{C}=\right.$ Curie temperature), where the system has lost all order in the spin arrangement. Intermediate temperatures are at present not accessible by explicit DFT calculations as prohibitively many large supercells would be needed to properly capture the partially destroyed long-range order and the inhomogeneously scattered regions with short-range order.

In the present Letter we therefore develop and apply a method that employs a set of DFT calculations carried out for only the FM and PM limits in conjunction with quantum Monte Carlo sampling to obtain properties at intermediate temperatures. The method is capable of describing magnonphonon coupling at arbitrary finite temperatures, closing the previous simulation gap between the $T=0 \mathrm{~K}$ and infinitetemperature limits. We demonstrate the performance of the method through good agreement of the calculated temperature dependence of the phonon frequencies in bcc Fe with experimental measurements including our new set of phonon frequencies measured at 38 temperatures. 


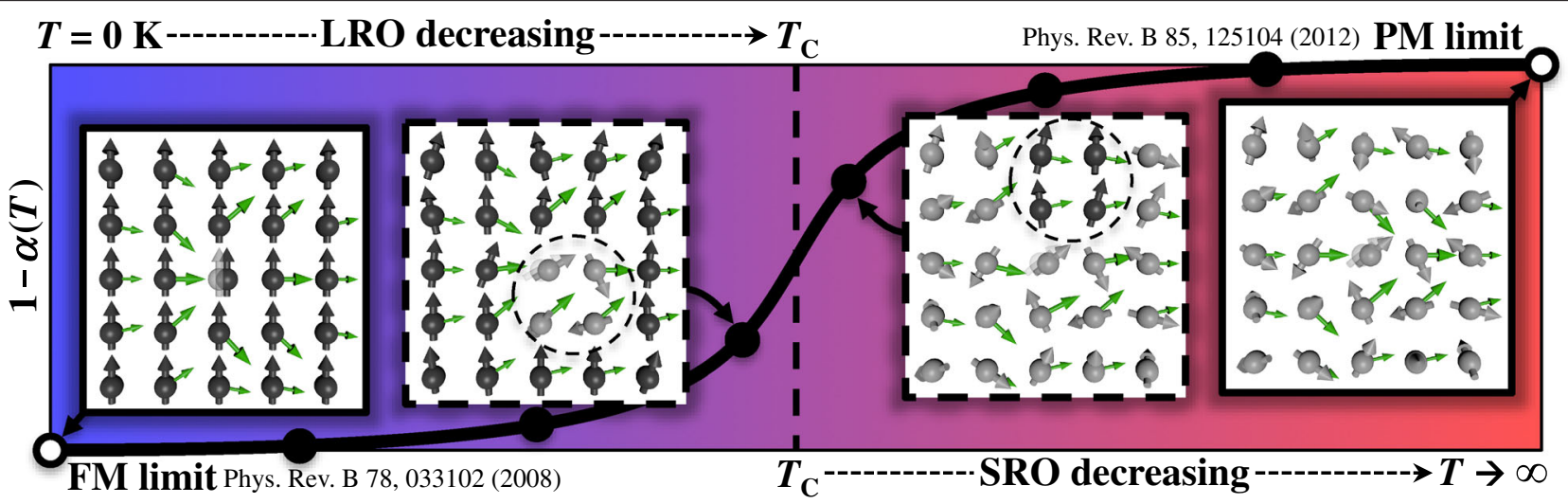

FIG. 1 (color online). Sketch of the developed method coupling the energetics of the magnetic system with explicit DFT force constant calculations (displaced spin in the middle of each sketch). The gray and green arrows indicate the local magnetic spins for each atom and the interatomic forces (restoring forces when displacing the center atom), respectively. The explicit force constant calculations are carried out for the two limits of completely ordered [17] (left side) and fully disordered spins [16] (right side). The force constants for the intermediate temperature regime (dashed squares) are obtained by coupling the two limits with quantum Monte Carlo simulations for the magnetic subsystem [19] which explicitly include long- and short-range-order effects (see text for details).

The basis of our method is the spin-space averaging (SSA) formalism within constrained-spin DFT [16]. Its starting point is the general magnetic Born-Oppenheimer energy surface $E_{\left\{\mathbf{s}_{i}\right\}}\left(\left\{\mathbf{R}_{i}\right\}\right)$ for a given set of atomic coordinates $\left\{\mathbf{R}_{i}\right\}$ and local magnetic spins $\left\{\mathbf{s}_{i}\right\}$, with $i$ running over all atoms. The corresponding SSA free energy and partition function are

$$
\mathcal{F}^{\mathrm{SSA}}(T)=-k_{B} T \ln Z, \quad Z=\sum_{\left\{\mathbf{s}_{i}\right\}} \exp \left[-\frac{E_{\left\{\mathbf{s}_{i}\right\}}}{k_{B} T}\right],
$$

where $k_{B}$ is the Boltzmann constant and where the sum extends over all magnetic configurations, assuming that the magnetic fluctuations are fast compared to the nuclear motion. The SSA force on atom $j$ is directly accessible from the gradient of the free energy as

$\mathbf{F}_{j}^{\mathrm{SSA}}(T)=-\partial \mathcal{F}^{\mathrm{SSA}}(T) / \partial \mathbf{R}_{j}=\sum_{\left\{\mathbf{s}_{i}\right\}} p_{\left\{\mathbf{s}_{i}\right\}}(T) \mathbf{F}_{j}\left(\left\{\mathbf{s}_{i}\right\}\right)$,

where

$$
p_{\left\{\mathbf{s}_{i}\right\}}(T)=\exp \left[-\frac{E_{\left\{\mathbf{s}_{i}\right\}}}{k_{B} T}\right] / Z
$$

is a Boltzmann weighting factor and

$$
\mathbf{F}_{j}\left(\left\{\mathbf{s}_{i}\right\}\right)=-\partial E_{\left\{\mathbf{s}_{i}\right\}} / \partial \mathbf{R}_{j}
$$

is the force for the atomic and magnetic configuration $\left(\left\{\mathbf{R}_{i}\right\},\left\{\mathbf{s}_{i}\right\}\right)$. In principle, the SSA formalism allows for the evaluation of interatomic forces at any given temperature if the weights and forces given by Eqs. (3) and (4) can be calculated. However, a calculation of Eqs. (3) and (4) employing explicit DFT calculations is presently not computationally feasible. Monte Carlo simulations and effective magnetic spin Hamiltonians (e.g., Refs. $[19,20]$ ) can be used to reduce the computational time by restricting the sampling of the Boltzmann weights to the most relevant configurations, but the remaining computations are still formidable. This has so far prevented any practical simulation of the full temperature-dependent magnon-phonon excitations.

We now develop an alternative approach to solve Eq. (2) efficiently. Our approach obtains the full temperature dependence of magnetic interatomic forces with explicit force calculations for only the two limiting cases of fully ordered $(T=0 \mathrm{~K})$ and fully disordered $(T \rightarrow \infty)$ magnetic configurations. Our key approximation is to expand the temperature-dependent many-body wave function in a reduced 2 -states basis of these two limits

$$
\begin{aligned}
\Psi(T)= & \sum_{\left\{\mathbf{s}_{i}\right\}} \sqrt{p_{\left\{\mathbf{s}_{i}\right\}}(T)} \Psi^{\left\{\mathbf{s}_{i}\right\}} \approx \sqrt{\alpha(T)} \Psi^{\mathrm{FM}} \\
& +\sqrt{1-\alpha(T)} \Psi^{\mathrm{PM}}
\end{aligned}
$$

where $p_{\left\{\mathbf{s}_{i}\right\}}(T)$ are the weights given in Eq. (3) and $\alpha(T)$ can be regarded as an adiabatic mixing parameter between both states (FM and PM). Using the expansion Eq. (5) in combination with the Hellmann-Feynman theorem [21], we obtain the following expression for the temperature dependent interatomic forces:

$$
\mathbf{F}_{j}^{\mathrm{SSA}}(T) \approx \alpha(T) \mathbf{F}_{j}^{\mathrm{FM}}+[1-\alpha(T)] \mathbf{F}_{j}^{\mathrm{PM}},
$$

where

$$
\mathbf{F}_{j}^{\mathrm{FM}}=-\partial E^{\mathrm{FM}} / \partial \mathbf{R}_{j}, \quad \mathbf{F}_{j}^{\mathrm{PM}}=-\partial E^{\mathrm{PM}} / \partial \mathbf{R}_{j} .
$$

The parameter $\alpha(T)$ can be obtained via the SSA internal energy, which can be formally decomposed as 
$E^{\mathrm{SSA}}(T)=\sum_{\left\{\mathbf{s}_{i}\right\}} p_{\left\{\mathbf{s}_{i}\right\}}(T) E_{\left\{\mathbf{s}_{i}\right\}} \approx \alpha(T) E^{\mathrm{FM}}+[1-\alpha(T)] E^{\mathrm{PM}}$,

with the energies of the two limits given by

$$
E^{\mathrm{FM}}=E^{\mathrm{SSA}}(T=0 \mathrm{~K}), \quad E^{\mathrm{PM}}=E^{\mathrm{SSA}}(T \rightarrow \infty) .
$$

If one chooses $\alpha(T)$ in Eq. (5) as

$$
\alpha(T)=\left[E^{\mathrm{SSA}}(T)-E^{\mathrm{PM}}\right]\left[E^{\mathrm{FM}}-E^{\mathrm{PM}}\right]^{-1},
$$

then Eq. (8) is formally exact.

The formulation derived here has two major advantages: (a) The forces in the FM and PM limit, $\mathbf{F}_{j}^{\mathrm{FM}}$ and $\mathbf{F}_{j}^{\mathrm{PM}}$, are fully defined by a gradient on the energy surface [Eq. (7)] rather than on the free energy surface. Computing the free energy surface would necessitate including a thermodynamic average over all magnetic states. While there is just one relevant configuration for the FM limit, the forces in the PM limit are governed by completely random spin configurations that are statistically degenerate [16]. (b) The temperature dependence is fully carried by the adiabatic coupling parameter $\alpha=\alpha(T)$. The explicit temperature dependence of this coupling parameter can be obtained independently of the DFT force calculations, e.g., as discussed below, via numerically exact quantum Monte Carlo simulations for an effective nearest-neighbor Heisenberg model [19].

We demonstrate the performance of our approach by applying the method to bcc Fe which is ferromagnetic at low temperatures and paramagnetic above the Curie temperature of $T_{C}=1043 \mathrm{~K}$. The interatomic forces required for calculating $\mathbf{F}_{j}^{\mathrm{FM}}$ and $\mathbf{F}_{j}^{\mathrm{PM}}$ in Eq. (7) have been computed in a $3 \times 3 \times 3$ cubic supercell containing 54 atoms as described in detail in Refs. [16,17]. We calculated the energies entering the coupling parameter $\alpha(T)$ in Ref. [19] by utilizing quantum Monte Carlo simulations [22-24] for an effective next-nearest-neighbor Heisenberg model in a supercell containing $\approx 10000$ spins. The simulations have been performed based on the direct loops algorithm in the stochastic series-expansion technique, a widely used approach for Heisenberg-like spin Hamiltonians (see, e.g., [24] and references therein).

The input parameters for the spin Hamiltonian are the theoretical $T_{C}$ [25] and the local magnetic spin moment (details of the method are given in Refs. $[19,26]$ ). The volume expansion was taken from experiment [27]. A typical temperature dependence of $1-\alpha(T)$ is sketched in Fig. 1 (thick solid line). The open circle in the lower left part refers to full ferromagnetic saturation (denoted as FM limit) and corresponds to a value of $\alpha(T=0 \mathrm{~K})=1$, whereas the case of fully disordered spins (i.e., complete absence of long-and short-range order) shown in the upper right part of the figure (PM limit) corresponds to a value of $\alpha(T \rightarrow \infty)=0$. The curvature of $\alpha(T)$ for intermediate temperatures is directly related to the well-known lambda-shaped magnetic contribution to the specific heat capacity [10].

In Fig. 2 we compare our computed phonon dispersion (red to orange solid lines in the right panel) with the neutron scattering experiments (filled circles) performed in Ref. [11]. The results correspond to three different temperatures 773,1043 , and $1173 \mathrm{~K}$ representing the relevant magnetic scenarios: $T<T_{C}, T=T_{C}$, and $T>T_{C}$. The highest temperature is just below the bcc to fcc transition at $1185 \mathrm{~K}$. The light-gray shaded area indicates the difference between the two limits of completely ordered (FM) and completely disordered (PM) magnetic spins. The largest impact of magnetic order (i.e., largest light-gray area) is observed for the energetically lower transverse $N-\Gamma$ branch, consistent with previous findings [14-16]. The temperature dependent phonon spectra computed with the present method lie constantly in-between the two theoretical limits, i.e., always within the gray shaded area, and show in general a very good agreement with the experimental data. An important observation is the relatively large value of $\alpha\left(T=1.1 T_{C}\right)=0.39$ at the high temperature of $1173 \mathrm{~K}$. This means that the magnetic short-range order is significant even considerably above $T_{C}$ (see "SRO" sketch in Fig. 1). The fact that $\alpha$ is still far away from zero at this

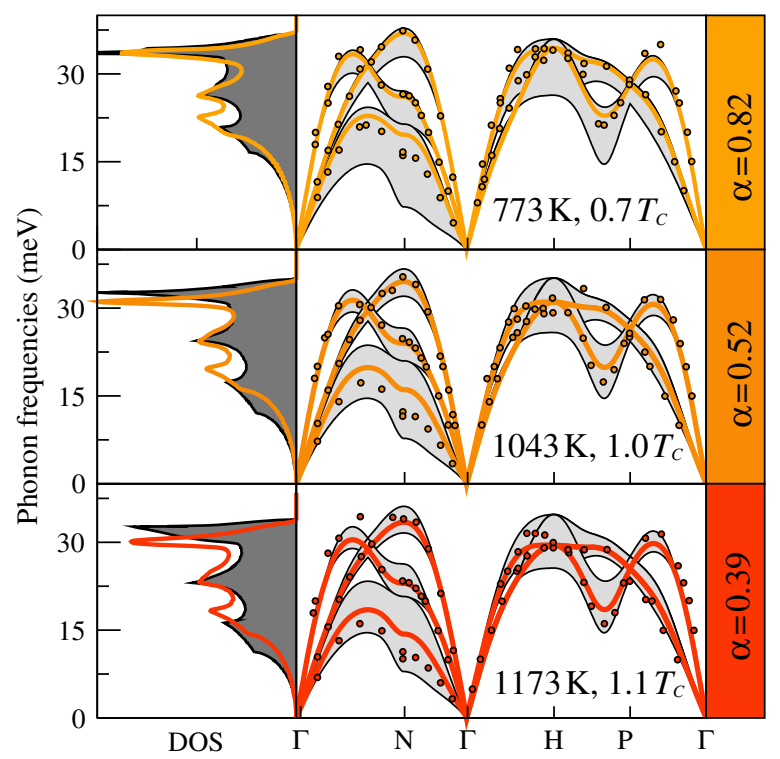

FIG. 2 (color online). Phonon density-of-states (DOS) and phonon dispersion of bcc $\mathrm{Fe}$ at three different temperatures. Symbols indicate experimental data obtained by neutron scattering experiments [11]. The light-gray shaded area within the phonon dispersion plots indicates the range between $\alpha=1$ (FM limit) and $\alpha=0$ (PM limit). The orange to red thick lines indicate the theoretical predictions for the corresponding $\alpha$ value (given to the right). The left panel shows the theoretical DOS (colored) and the DOS obtained by fitting the neutron spectra by a Born—von Kármán fit [28] (dark gray shaded area). 
temperature explains the unexpected observation that the temperature-dependent phonon spectrum at $1173 \mathrm{~K}$ (red lines) is still significantly larger than the one computed within the PM limit (lower black solid lines). The importance of magnetic short-range order on thermodynamic properties has been previously discussed [19] and is consistent with recent results obtained by Ruban and Razumovskiy [15] using the spin-spiral approach.

Comparing absolute phonon frequencies at a fixed temperature as in Fig. 2 is not very well suited for revealing the performance of our proposed methodology. The DFT itself has an inherent difficulty describing absolute phonon frequencies accurately due to the exchange-correlation approximation [29]. For example, for the PBE-GGA exchange-correlation functional used here, the $\Gamma-N$ transverse phonon branch is known to be overestimated compared to experimental data [17]. This deficiency is already apparent at $T=0 \mathrm{~K}$ and does not imply a shortcoming of the present methodology which focuses on the temperature dependence. We therefore compare the changes in phonon energy with temperature (relative to $T=0 \mathrm{~K}$ ) to the temperature trends in the experimentally observed phonon energies, rather than using the absolute values.

Figure 3 shows the comparison for a representative set of $q$ points and phonon branches. The neutron scattering experiments in Ref. [11] provide a detailed temperature dependence for two $q$ points. Our recently performed phonon density of states measurements have been analyzed by Born-von Kármán (BvK) analysis to provide more detailed temperature trends [12]. The optimized BvK parameters permit the calculation of phonon frequencies at any $q$ point. For branches where neutron-scattering experimental data are available (filled black squares), our

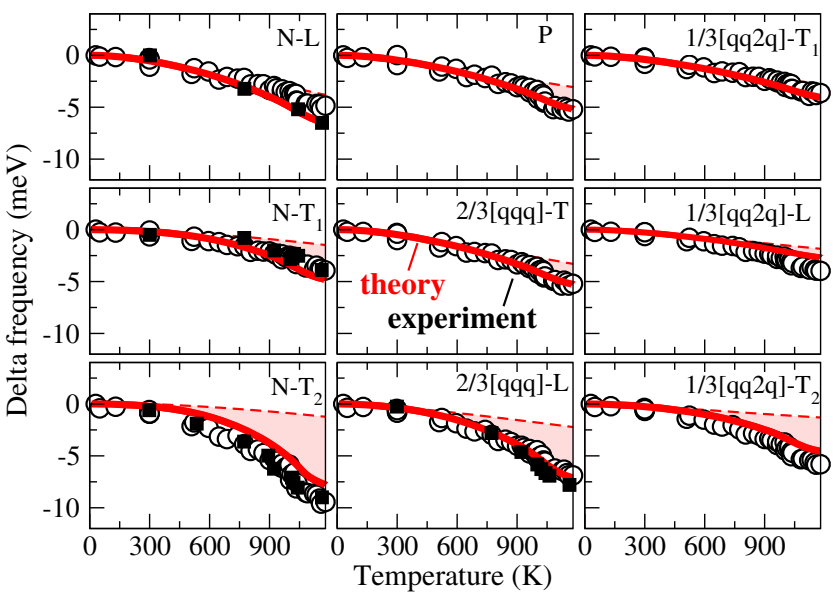

FIG. 3 (color online). Temperature dependence of phonon frequencies for different modes comparing theoretical (solid red lines) with experimentally deduced frequencies (open circles) [12]. Dashed red lines show the ferromagnetic quasiharmonic results (no phonon-magnon coupling). Where available, neutron scattering data from Ref. [11] are included (filled black squares). experimental $\mathrm{BvK}$ fitted frequencies (open black circles) agree well.

The temperature dependence of our theoretical calculations (red lines) is in excellent agreement with experiment in all cases. In addition to the complete theoretical temperature dependence, we also show the ferromagnetic frequencies (dashed red lines) which inherit their temperature dependence from the quasiharmonic approximation. In some cases (e.g., for the $1 / 3$ [qq $2 \mathrm{q}] T_{1}$ point) the temperature dependence is dominated by the quasiharmonic changes and magnon-phonon coupling is negligible. For other regions (e.g., $\mathrm{N}-\mathrm{T}_{2}$ point) the magnon-phonon coupling is strong, in fact much stronger than the quasiharmonic dependence, demonstrating that employing the introduced coupling scheme is indispensable. The large softening of these modes is directly linked to a strong decrease in the elastic constants, which are involved in the structural transformation path from bcc to fcc $[14,16]$. As mentioned above, experimental analysis suggests explicit anharmonic contributions to be an order of magnitude smaller compared to the observed phonon softening [12]. Nevertheless, explicit DFT studies including anharmonic and magnetic effects, such as DLM molecular dynamics, are attractive but so far lacking for bcc $\mathrm{Fe}$. A recent application to $\mathrm{CrN}$ revealed a good agreement between SSA and DLM molecular dynamics [30,31].

In conclusion, we developed an efficient and accurate method to compute interatomic forces at arbitrary magnetic temperatures. The method couples the recently proposed spin-space averaging formalism with a quantum mechanical spin Hamiltonian for the magnetic subsystem. We showed that the energetics and temperature dependence of the magnetic subsystem can be decoupled from the much more computationally demanding DFT force constant calculations. Combining numerically exact quantum Monte Carlo simulations for the magnetic spin system with highly accurate force constant calculations, we find an excellent agreement between theory and a large set of experimental data for the temperature dependence of phonon frequencies in bcc Fe. If applied to more complex structures, the limitation of the explicit force computations to two magnetic states (the ferro-and paramagnetic configurations) might be insufficient. Specifically, an extension would be required in systems with locally preserved magnetic order above the global magnetic transition temperature (e.g., within extended defects). Finally, we note that the method developed here to investigate effects of magnon-phonon coupling is not restricted to bcc Fe. It will be interesting to apply it to other magnetic structures (e.g., antiferromagnets) or more complex magnetic alloys, and to compute thermodynamic properties such as, e.g., magnon-phonon contributions to thermal expansion. It is appealing to apply similar strategies to investigate the impact of substitutional SRO on the atomic motion by coupling, e.g., energetics of Monte Carlo-cluster expansion techniques with force computations of chemically ordered and disordered alloys. 
Funding by the Deutsche Forschungsgemeinschaft (DFG) within the collaborative research center SFB 761 "Stahl- $a b$ initio" and within the priority program SPP 1599, and by the European Research Council under the EU's 7th Framework Programme (No. FP7/2007-2013)/ ERC Grant Agreement No. 290998 is gratefully acknowledged. L. M. and B.F. acknowledge support of DOENNSA Award No. DE-NA0001974 and DOE-BES Award No. DE-FG02-99ER45775.

[1] P. Steneteg, B. Alling, and I. A. Abrikosov, Phys. Rev. B 85, 144404 (2012).

[2] S. Dudarev and P. Derlet, J. Phys. Condens. Matter 17, 7097 (2005).

[3] T. Hickel, M. Uijttewaal, A. Al-Zubi, B. Dutta, B. Grabowski, and J. Neugebauer, Adv. Eng. Mater. 14, 547 (2012).

[4] R. C. Reed, The Superalloys: Fundamentals and Applications (Cambridge University Press, Cambridge, England, 2006).

[5] T. Hickel, B. Grabowski, F. Körmann, and J. Neugebauer, J. Phys. Condens. Matter 24, 053202 (2012).

[6] C. M. Fang, M. H. F. Sluiter, M. A. van Huis, C. K. Ande, and H. W. Zandbergen, Phys. Rev. Lett. 105, 055503 (2010).

[7] M. van Schilfgaarde, I. Abrikosov, and B. Johansson, Nature (London) 400, 46 (1999).

[8] H. Ding, V. I. Razumovskiy, and M. Asta, Acta Mater. 70, 130 (2014).

[9] S. L. Dudarev, R. Bullough, and P. M. Derlet, Phys. Rev. Lett. 100, 135503 (2008).

[10] F. Körmann, A. A. H. Breidi, S. L. Dudarev, N. Dupin, G. Ghosh, T. Hickel, P. Korzhavyi, J. A. Muñoz, and I. Ohnuma, Phys. Status Solidi B 251, 53 (2014).

[11] J. Neuhaus, W. Petry, and A. Krimmel, Physica (Amsterdam) 234B, 897 (1997).

[12] L. Mauger, M. S. Lucas, J. A. Muñoz, S. J. Tracy, M. Kresch, Y. Xiao, P. Chow, and B. Fultz, Phys. Rev. B 90, 064303 (2014).

[13] S. Baroni, S. de Gironcoli, A. Dal Corso, and P. Giannozzi, Rev. Mod. Phys. 73, 515 (2001).

[14] I. Leonov, A. I. Poteryaev, V. I. Anisimov, and D. Vollhardt, Phys. Rev. B 85, 020401 (2012).

[15] A. V. Ruban and V. I. Razumovskiy, Phys. Rev. B 85, 174407 (2012).
[16] F. Körmann, A. Dick, B. Grabowski, T. Hickel, and J. Neugebauer, Phys. Rev. B 85, 125104 (2012).

[17] F. Körmann, A. Dick, B. Grabowski, B. Hallstedt, T. Hickel, and J. Neugebauer, Phys. Rev. B 78, 033102 (2008).

[18] D. Perera, D. P. Landau, D. M. Nicholson, G. M. Stocks, M. Eisenbach, J. Yin, and G. Brown, J. Appl. Phys. 115, 17D124 (2014).

[19] F. Körmann, A. Dick, T. Hickel, and J. Neugebauer, Phys. Rev. B 83, 165114 (2011).

[20] F. Körmann, A. Dick, T. Hickel, and J. Neugebauer, Phys. Rev. B 81, 134425 (2010).

[21] J. Harris, R. Jones, and J. Müller, J. Chem. Phys. 75, 3904 (1981).

[22] F. Alet, S. Wessel, and M. Troyer, Phys. Rev. E 71, 036706 (2005).

[23] A. Albuquerque, F. Alet, P. Corboz, P. Dayal, A. Feiguin, S. Fuchs, L. Gamper, E. Gull, S. Gürtler, A. Honecker et al., J. Magn. Magn. Mater. 310, 1187 (2007).

[24] B. Bauer, L. Carr, H. G. Evertz, A. Feiguin, J. Freire, S. Fuchs, L. Gamper, J. Gukelberger, E. Gull, S. Guertler et al., J. Stat. Mech. (2011) P05001.

[25] F. Körmann, A. Dick, T. Hickel, and J. Neugebauer, Phys. Rev. B 79, 184406 (2009).

[26] F. Körmann, B. Grabowski, P. Söderlind, M. Palumbo, S. Fries, T. Hickel, and J. Neugebauer, J. Phys. Condens. Matter 25, 425401 (2013).

[27] The experimental relative lattice expansion $a^{\exp }(T) / a^{\exp }(0)$ [Y.S. Touloukian, Thermal Expansion: Thermophysical Properties of Matter (Plenum Press, New York, 1975), Vol. 12] has been combined with the theoretical value of $a^{\text {theo }}(0)=2.83$ A. The actual force calculations for FM and PM state were performed for 4 volumes in the considered range. A 2nd order polynomial fit has been employed to parametrize the volume dependence of the interatomic force constants afterwards.

[28] J. Neuhaus, M. Leitner, K. Nicolaus, W. Petry, B. Hennion, and A. Hiess, Phys. Rev. B 89, 184302 (2014).

[29] B. Grabowski, T. Hickel, and J. Neugebauer, Phys. Rev. B 76, 024309 (2007).

[30] N. Shulumba, B. Alling, O. Hellman, E. Mozafari, P. Steneteg, M. Odén, and I. A. Abrikosov, Phys. Rev. B 89, 174108 (2014).

[31] L. Zhou, D. Holec, M. Bartosik, F. Körmann, B. Grabowski, J. Neugebauer, and P. H. Mayrhofer, arXiv:1408.3201. 